Bella tacenda parant; nullo iam noxia partu

30 Femina quaecumque est hostem parit; arua iuuentus

Nuda fodit tardoque puer domi fultus aratro

Miratur patriis pendentem sedibus ensem.

sed procul a nobis infelix gloria syllae

Tetraque tempestas Marii, cum Roma supremas

35 Desperauit ... . et Martia uendidit arma.

Nunc tellus inculta nouos parit ubere fetus,

Nunc ratibus tutis fera nou irascitur unda,

Mordent frena tigres, subient iuga saeua leones,

Casta faue Lucina, tuus iam regnat Apollo.

FINIT.

Bern.

terrat

H. Hagen.

31 domi factus $\quad 32$ sedibus ensem $\quad 33$ sillę $\quad 34$ Trinaque tempesta moriens cum roma supremas desperabit et martia 36 foetus 38 tygres sęua 39 Casta faue etc.] $=$ Verg. Eel. IV, 10. leberhaupt diente dem dichter Vergil's vierte ecloge sum vorbild.

\title{
B. Zur erklärung und kritik der schriftsteller.
}

\section{Zu Sophocles.}

Die ausgaben von Brunck und Schneidewin bieten Trach. 1019 folgende lesart:

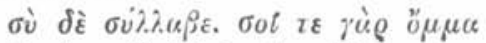

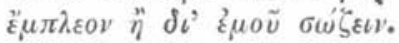

Es sind dies die worte, die der greis an den Hyllos richtet. Die schwierigkeit liegt meiner meinung nach durchaus nicht in ǒ $\mu \mu c$; Schneidewius frage, wozu es hier der augen bedarf, wird unten beantwortet werden. Zunäichst halte ich $\xi^{\prime} \mu \pi \lambda$ kov $\ddot{\eta}$ für falsch und

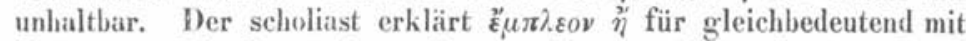

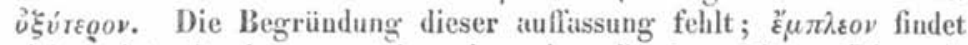

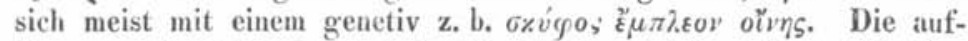
fassung ,ein volles auge $d$. h. ein auge, welches die vollständige sehkraft hat", ist gesucht und ohne analogie. Der ausdruck, plenum lumen" bei Claudian, de raptu Proserpinae III, 441, auf den man sich beziehen könnte, besagt nicht, dass das auge der die tochter suchenden Ceres scharf war, sondern aufmerksam und gespannt. Zugegeben auch, dass $\tilde{\varepsilon}^{\prime} \mu \pi \lambda \lambda_{\varepsilon o v}$ die bedeutung von $\partial \grave{b} \xi \grave{v}$ usurpiren kann, so ist die schwierigkeit der stelle noch gar nicht gelöst. Das $\ddot{\eta}$ nach dem positiv lässt sich nicht vertheidigen. Kiihner ?. 624, 2 findet hier keine anwendung. Der Grieche hat ferner mit $\not$ nach comparativen bestimmte prapositions-verbinduu-

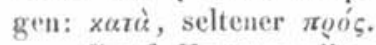

Gottf. Hermann die schwierigkeiten uicht übersehend, welche dic stelle in sprachlicher hinsicht bietet, schlug folgendes vor: 


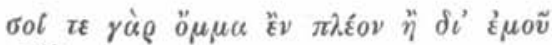

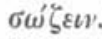

,Tibi plena est atque integra videndi facultas, potius quam ut per me ille servari possil". Der an und für sich richtige gedanke wird aus den dasteheuden worten nicht eruirt werden können. Auch an dieser conjectur lisst manches sich ausstellen, was oben bereits erwähnt worden ist; die ganze ausdrucksweise ist viel zu brachylogisch. S'chwer ist es jedenfalls das

Döderlein schlägt im Philol. X, p. 334 vor:

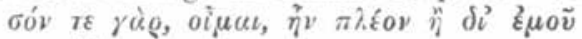

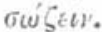

Abgesehen von $\delta$ ' żuov als ungewöhnlicher sprachweise nach comparativ mit $\ddot{\eta}$ ist von Nauck mit recht die verwechslung des $\pi \lambda \varepsilon_{0 \nu}$ mit $\mu \tilde{u} \lambda \lambda . \nu \nu$ gerügt worden. Weit unerträglicher ist der sinn der stelle in dieser form. „Es war mehr deine pflicht, glaube ich, durch dich als durch mich ilın zu retten". Es war - ist es denn jetzt nicht melır? Wozu fordert denn der schmerzgepeinigte Herkules auf mit den worten $\sigma \dot{u} \lambda . \alpha_{\beta} \varepsilon$ ? Soll aber in den worten ein vorwurf gegen Hyllos liegen, dass er bis jetzt mit dem vater sich nicht beschäftigt habe, so sieht man nicht ein, mit welchem recht dieser vorwurf gegen Hyllos geschleudert ist. Ausserdem involvirte ein solcher vorwurf die niedrigste gesinnung des greises, der zu viel für seitsen herrn gethan zu haben glaubt, eine gesinnung, deren gegentheil im stücke überall manifestirt ist. Im übrigen liesse der brachylogische ausdruck noch immer eine klippe von schwierigkeit zurück.

Wakefields conjectur, die Erfurdt in den text aufgenommeu hat, oi $\mu c$, ist ebenfalls schwer und unhaltbar. oìc bedeutet, angriff" und wird gewöhnlich von löwen und raubvögeln gebraucht. Ob man es von der krankheit sagen kann, ist eine frage, die keine analoge stelle günstig beantwortet. Hier aber, wo oîu absolut steht, ist es gar nicht zu halten. Was soll denn ferner otuu

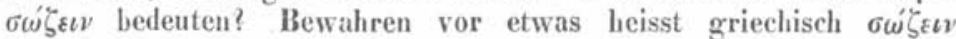

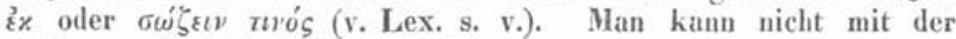
stelle aus Euripides Phoen. 600 kommen

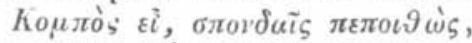

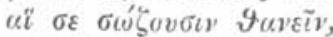

um die verbindung $\sigma \omega \omega^{\zeta} \varepsilon \iota v$ oicuc zu rechtfertigen. Der infinitiv $\vartheta \varkappa r \varepsilon \tilde{\imath}$ ist expletiver natur und könnte auch füglich fehlen. Ausserdem ist bei Euripides dits persönliche object $\sigma \varepsilon$ zu lesen. Schon mit rücksicht auf diese schwierigkeit allein ist die Wakefieldsche conjectur eine verunglïckte zu nenuen.

Ich komme nun zu meiner eigeneu. Sie lautet:

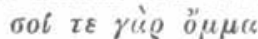

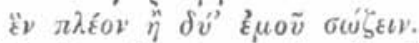

Dass sich diese lesart aus der überlieferten, corrumpirten mit gro- 
sser leichtigkeit herstellen lässt, bedarf nicht weiterer auseinandersetzung. Wie steht es mit dem sinn der stelle? Wörtlich übersetzt heisst die stelle nach meiner schreibweise: ,dir ist ein auge melir als zwei von mir zu helfen" d. h. du vermagst mit einem auge ihm melır zu dienen, als ich mit zweien. Was bedarf es des auges? Herkules empfindet schmerz am körper; eine unrechte berïhrung kann den von brandwunden erzeugten schmerz nur noch vergrössern, deshalb ist vorsicht nöthig, die der alte bei dem besten willen nicht mit günstigem erfolg anwenden kann, da ihm

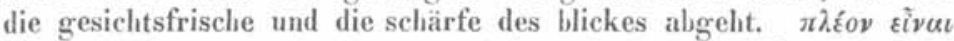
lieisst hier, was es iiberhaupt nur heissen kann, plus efficere. Aehnliche verbindung findet sich bei Xenoph. Cyrop. 5. 5. 34. Soph. Ant. 268.

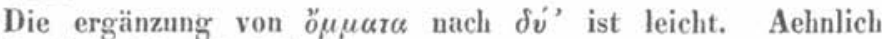
schreilst Ovid. Met. 4, 158.

At tu, quae ramis arbor miserabile corpus

Nunc tegis unius, mox es tectura duorum.

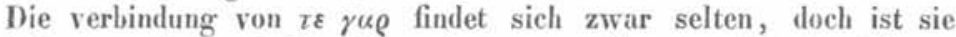
kein grund zur bekämpfung der vorgeschlagenen lesart. Das $\tau \varepsilon$ verbindet hier nicht, wie Hermann treflend bemerkt, sondern bekräftigt die worte im milderen maasse als $\tau o \iota$, wie es sich oft bei Homer nach relativis findet $E$. 467. Aehnliche verbindungen sind

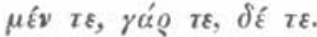

Posen.

Herrmann Warschauer.

\section{Zu Sophokles.}

Die annahme einer tragödie des Sophokles ' $Q \varrho \varepsilon \imath \vartheta v \iota \alpha$ (frg.

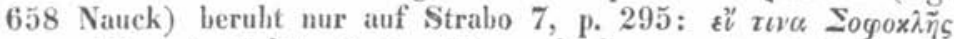

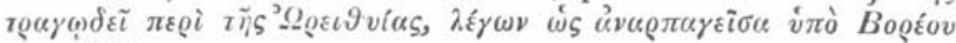

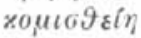

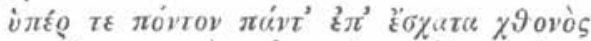

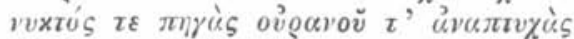

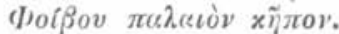

Diese stelle gehört aber, wie es mir scheint, zu den tympanisten, welche die sage von den söhnen der Kleopatra behandelten nach

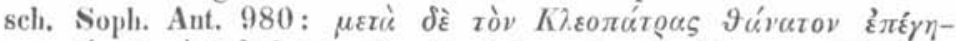

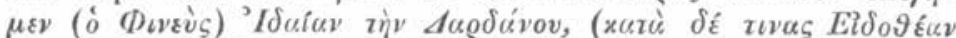

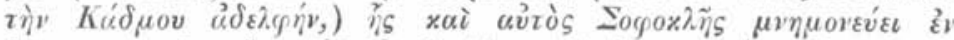

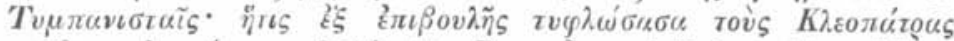

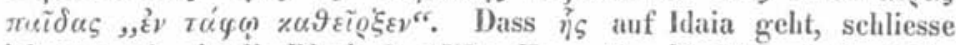

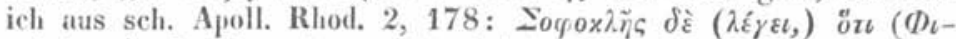

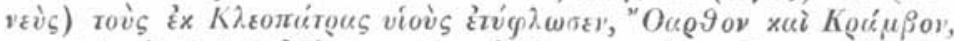

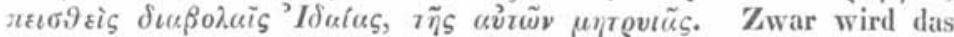
vvghioṽ einmal dem Phineus, das andre mal der Idaia zugeschrie- 